\title{
Blocking microglial proliferation halts Alzheimer disease in mice
}

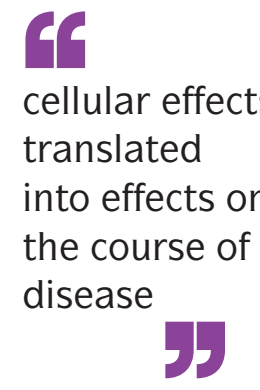

Targeting of a pathway that controls microglial proliferation could offer a novel therapeutic approach to Alzheimer disease (AD), according to recent research. In a mouse model of $\mathrm{AD}$, inhibition of colony-stimulating factor 1 receptor (CSF1R) improved memory and prevented synaptic degeneration.

Accumulating evidence suggests that proliferation of microglia has an important role in neurodegeneration. Previous research by Diego GomezNicola and colleagues has focused on a mouse model of prion disease and demonstrated that activation of CSF1R is important in controlling proliferation of microglia. "A significant body of knowledge suggests that CSF1R also plays a role in controlling microglial function in $\mathrm{AD}$, so we

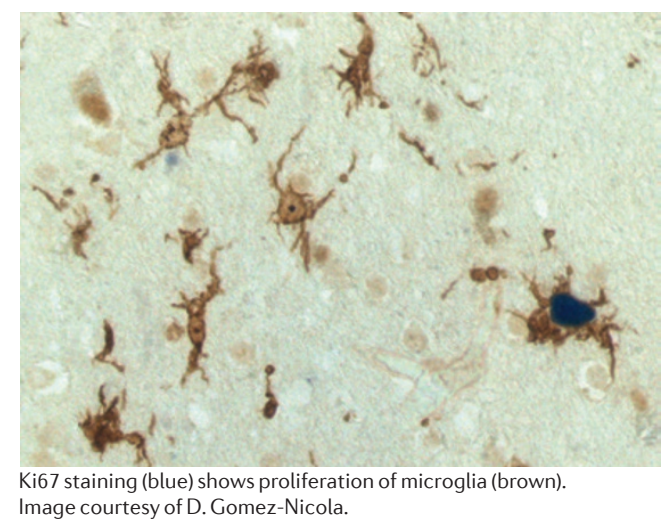

decided to evaluate this hypothesis," explains Gomez-Nicola.

The researchers first confirmed that microglial proliferation is a characteristic of $\mathrm{AD}$ in humans by analysing 10 post-mortem brain samples from patients with $\mathrm{AD}$. The proportion of microglia that expressed Ki67 - a marker of proliferation - in these samples was more than twice that in samples from healthy controls. Components of the CSF1R pathway were also upregulated in patients with $\mathrm{AD}$, indicating that the increased microglia proliferation is the result of CSF1R activation.

Gomez-Nicola and colleagues then confirmed that CSF1R-controlled microglial proliferation was elevated in APP/PS1 mice, which exhibit AD-like pathology, before assessing the effects of blocking CSF1R. They showed that prolonged treatment with the CSF1R inhibitor GW2580 blocked proliferation of microglia, downregulated genes associated with the CSF1R pathway, and reduced expression of several inflammatory mediators.

These cellular effects translated into effects on the course of disease. The treatment prevented development of behavioural deficits, and improved short-term memory and synaptic density in the hippocampus.
The number of amyloid plaques, however, was unaffected, adding to existing evidence that amyloid pathology does not directly contribute to $\mathrm{AD}$ progression.

"Our findings support existing clinical and genome-wide association studies that showed a correlation between brain inflammation and $\mathrm{AD}$ progression, but we provide a mechanistic insight," explains Gomez Nicola. "We have also highlighted the relevance of CSF1R in $\mathrm{AD}$, and suggest its targeting as a promising therapeutic approach."

The authors warn that use of CSF1R inhibitors as therapeutic agents will require a cautious approach owing to possible adverse effects. Nevertheless, they have already undertaken initiatives with pharmaceutical companies to exploit the full clinical potential of their findings.

"These initiatives have a clearly defined translational mindset, and we are now aiming to convert our current findings into benefits for $\mathrm{AD}$ patients as soon as possible," says Gomez-Nicola.

Ian Fyfe

ORIGINAL ARTICLE Olmos-Alonso, A. et al. Pharmacological targeting of CSFR1R inhibits microglial proliferation and prevents the progression of Alzheimer's-like pathology. Brain, http://dx.doi.org/10.1093/brain/awv379 